\title{
Building profile reconstruction using TerraSAR-X data time-series and tomographic techniques
}

\author{
Martina Porfiri* ${ }^{\dagger}$, Laurent Ferro-Famil ${ }^{*}$ and Jean-Marie Nicolas ${ }^{\ddagger}$ \\ *University of Rennes 1, IETR, Remote Sensing Group, Rennes, France \\ Email: (laurent.ferro-famil, martina.porfiri)@univ-rennes1.fr \\ †University of Rome 'La Sapienza', DICEA, Geodesy and Geomatics Division, Rome, Italy \\ Email: martina.porfiri@uniroma1.it \\ †Institut Mines-Telecom, Telecom ParisTech, CNRS LTCI, Paris, France \\ Email: jean-marie.nicolas@telecom-paristech.fr
}

\begin{abstract}
This work aims to show the potentialities of SAR Tomography (TomoSAR) techniques for the 3-D characterization (height, reflectivity, time stability) of built-up areas using data acquired by the satellite sensor TerraSAR-X. For this purpose 19 TerraSAR-X single-polarimetric multibaseline images acquired over Paris urban area have been processed applying classical nonparametric (Beamforming and Capon) and parametric (MUSIC) spectral estimation techniques.
\end{abstract}

\section{INTRODUCTION}

The Earth is a dynamic system and its surface is continuously changing at different time scales. Satellite multitemporal imagery and derived secondary products play a relevant role in supplying the information necessary for anlysing, monitoring and characterizing the Earth's surface dynamics. Thanks to the recent SAR (Synthetic Aperture Radar) satellite missions as TerraSAR-X and CosmoSkymed, it is possible to achieve very high spatial and temporal resolution, requirements of essential importance for tridimensional SAR imaging techniques, such as SAR Tomography (TomoSAR).

This technique, firstly introduced in [1], is based on the use of more than two SAR acquisitions performed in interferometric configuration and combined in order to form an additional synthetic aperture in the elevation direction achieving a substantially improved resolution. It is used to solve the scattering ambiguity problem in the vertical direction by separating multiple scatterers within the same resolution cell. In this way it may be possible to correct geometric distortions derived by the layover, a phenomenon that induces on the radar image the reverse ordering of surface elements respect to the ordering on the ground. Regarding high spatial resolution data, the impact of layover highly increases because it affects a larger number of image pixels [2] [3]. Moreover, for this kind of analysis an important (and challenging) requirement is a good correlation between data acquired over a large temporal span.

\section{SAR TOMOGRAPHY}

SAR Tomography is based on the acquisition of $M$ SAR signals along slightly shifted trajectories $t_{i}$. After focusing, compensating and georeferencing the acquired signals, $M 2 \mathrm{D}$ SAR images are obtained. Due to its intrinsic cylindrical ambiguity, classical 2D SAR imaging is not well adapted to the characterization of volumic media. The purpose of TomoSAR imaging is to improve the vertical resolution of the SAR measurements in order to estimate the reflectivity in a more accurate (3-D) way. To do so, all the combinations of two SAR images out of the $M$ acquired ones are used in the frame of a classical problem of spectral estimation (for which a series of solutions can be applied [4] [5] [6] [7]). SAR tomography can be derived from the data covariance matrix $\widehat{\mathbf{R}}$ using monoand multi- dimensional spectral estimations [8]. In this work the following mono-dimensional methods are used: the Beamforming and Capon, non-parametric approaches generally used to globally appreciate the structure of a volumetric medium; MUSIC, parametric approach generally characterized by better resolution and performance for the analysis of discrete sources and affected by data modelling errors.

\section{DATASET}

In order to perform the present work, a stack of 21 singlepolarimetric TerraSAR-X images acquired over Paris urban area have been considered. In Figure 1(a) and 1(b) the spatial baseline and the spatial-temporal baselines distributions are respectively shown; the elevation aperture size is about 583.5 $\mathrm{m}$ and the temporal baseline ranges from 24th January 2009 and 26th November 2010. From Figure 2 one may appreciate the distortions due to layover: taller buildings within the subset are 'lying down' onto the ground along the range direction, showing both their roof, façade and base and interfering with scatterers situated below them. In Figure 3(a) and 3(b) the

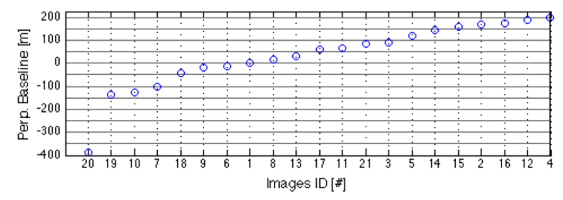

(a) Perpendicular baseline distibution

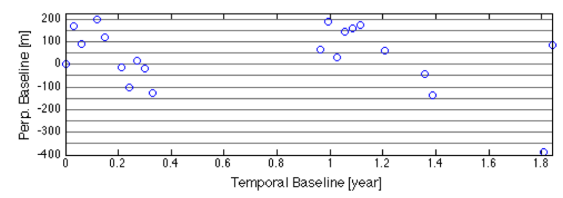

(b) Spatial-temporal baseline distribution

Figure 1. Distributions of the 21 acquisitions in spatial and temporal domains 


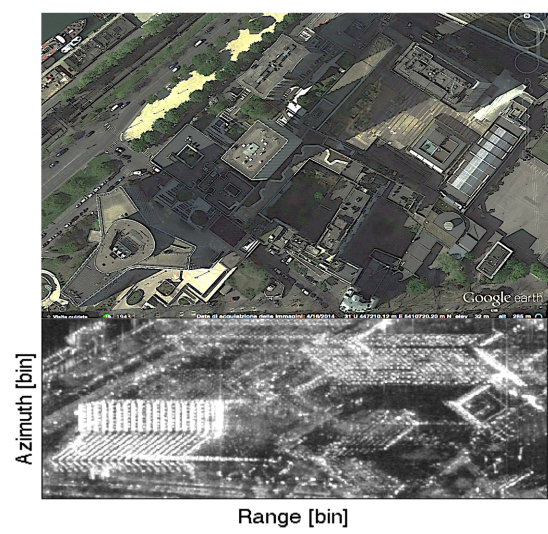

Figure 2. Image taken from Google Earth (top) and multitemporal averaged amplitude image derived from TerraSAR-X data stack (bottom)

calibrated coherence maps evaluated along both the increasing temporal and absolute spatial baselines are reported. In particular, amplitude images (principal diagonal), coherence images (upper triangular part) and flattened and calibrated interferograms (lower triangular part) computed over the entire processed stack are depicted. Both figures show the trend of the interferometric phase quality for all the combinations of two images out of the stack. For example, image with index equal to 20 results highly uncorrelated with all other images most probably because it presents both the higher spatial baseline value (about $-386.7 \mathrm{~m}$ ) and a dispersed acquisition date (see Figure 1(a) and 1(b)). On the other hand, pair images with small baselines are characterized by a higher correlation presenting better and brighter coherence images. In order to better appreciate the globally interferometric quality trend, in Figure 4 it is is represented the mean coherence values evaluated over several image subsets characterized by different scenes, i.e. layover, mixed and vegetated areas. It is evident how the coherence increases both moving from the top to the bottom and, especially for the layover area, decreasing the spatial baselines values.

On the basis of these preliminary analysis the two (temporally) last images have been discarded and the experimental results have been achieved processing the leftover 19 images.

\section{EXPERIMENTAL RESULTS}

\section{A. Building height estimation}

In order to retrieve buildings height information, SAR tomographic results has been achieved implementing and applying different mono-dimensional estimators, in particular the Beamforming, Capon and MUSIC ones. The present work was mainly focused on the 3-D SAR tomography technique, thus 4-D (differential, i.e. space-time) methods, as compressive sensing (CS) proposed in [9] [10], have not been taken into account.

Tomograms illustrated in Figure 5(b) are referred to the image profile highlighted in Figure 5(a) by the red line and strongly catheterized by the geometric distortions related to the layover. Moving from the top to the bottom one may note the effective vertical resolution improvement between the different approaches: sidelobes, probably due to atmospheric phase noise residuals, are particularly strong for Beamforming estimations, strongly reduced by Capon and completely

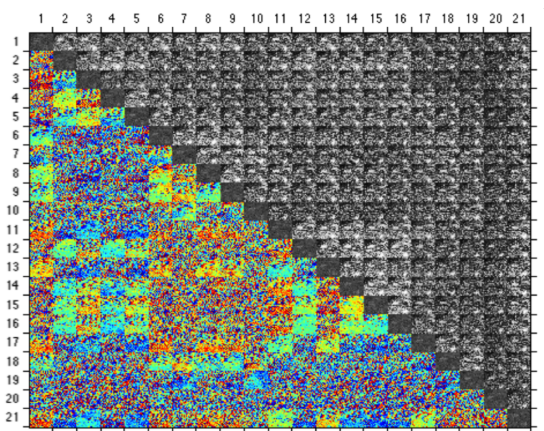

(a) Increasing temporal baseline

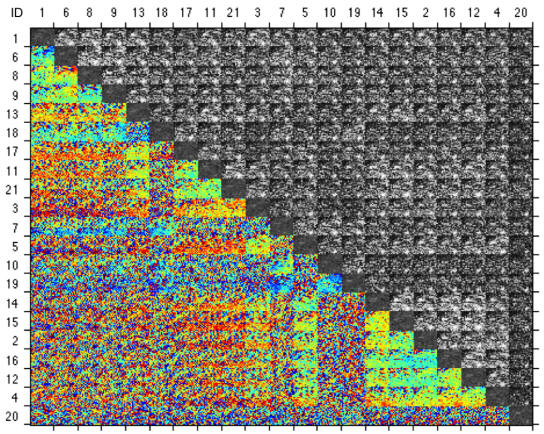

(b) Increasing spatial baseline

Figure 3. Calibrated coherence maps along temporal and spatial baselines

resolved by MUSIC approach. Thanks to the high quality resolution of the processed data, it is possible to distinguish, for some resolution cells, more than one intensity local maxima related to different scatters due mainly to the skyscrapers and the ground. These results demonstrate the possibility to resolve pixels affected by the layover, i.e distinguishing multiple scatterers within one resolution cell, using basic and classical spectral estimation models. In order to better investigate and appreciate the obtained results, a 3-D reconstruction has been performed over the analysed profile. The 3-D view has been reconstructed in ground range considering the results obtained from MUSIC approach and more than one scatters within one resolution cell. Indeed, with respect to Capon and Beamfoming ones, a higher number of points related to the second and third scatters (respectively about the $20 \%$ and $3 \%$ respect to the dominant ones) have been detected. From Figure 6(a), focusing on the biggest skyscraper, one may perceive its shape and the location of its two façades. Moreover, comparing with the

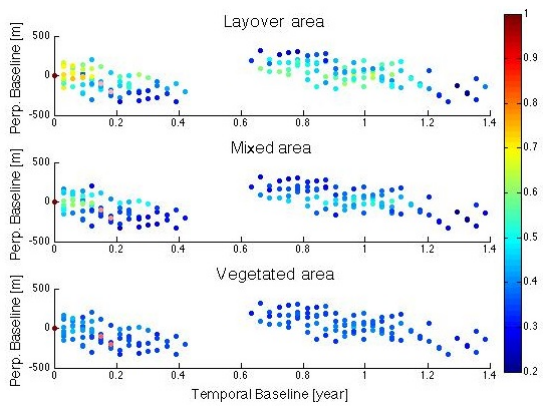

Figure 4. Spatial-temporal trend of coherence mean values evaluated over different image subsets 


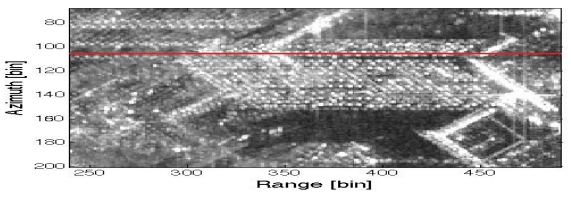

(a) Analysed profile highlighted in red over the AOI

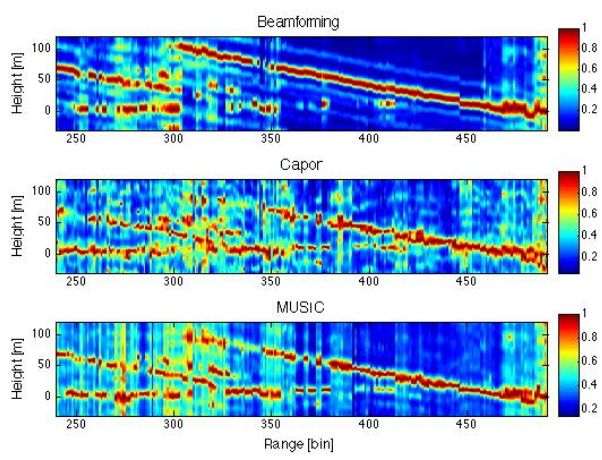

(b) Slant range normalised reflectivity tomograms

Figure 5. Tomograms generated over one profile applying Beamforming, Capon and MUSIC spectral estimators

amplitude image in Figure 2 (upper-right part), it is clear that resolution cells affected by layover have been georeferenced and the information related to features below them has been partially reconstructed in the others two main buildings and the ground, as shown in Figure 6(b). From the 3-D reflectivity map shown in Figure 6(c) it is evident the strong double bounce scattering mechanisms mainly related to the basis of the main buildings whereas the ground, accordingly with the expectations, is characterized by lower reflectivity values.

\section{B. Time series analysis}

In order to deeper characterize the studied area, a stationarity analysis has been assessed over the data stack by testing the fluctuation of the intensity of the signal [11]. The parameter $\Lambda$ has been computed applying the equation 1

$$
\Lambda=\frac{\prod_{i=1}^{N} \mathbf{I}_{i}^{\frac{1}{N}}}{\frac{1}{N} \sum_{i=1}^{N} \mathbf{I}_{i}}
$$

where $N$ is the images number and $\mathbf{I}_{i}$ represents the intensity matrix of the i-th image. The relative image over all Paris scene is represented in Figure 7: features like buildings and bridges show high $\Lambda$ values, whereas boats and main streets are affected by a very low stationary behaviour. Moreover, the phase coherence indicator trend computed according to [12] and depicted in Figure 8 shows the low cross-correlation of natural areas (e.g. the river in the upper right part of the images), against the higher values of the buildings (especially over the squared lower-left roof).

In order to assess the 3-D temporal stability of the observed scene, a set of 3-D intensities $I_{i}(x, y, z)$, with $i=1, \ldots, N_{i m}$, is built from $N_{i m}-12-\mathrm{D}$ images, $\left\{s_{j}\right\}_{j=1, j \neq i}^{N}$. These 3D intensity estimates have similar resolution properties, and variations with $i$ permit to appreciate the importance of the missing image. The temporal stability at coordinates $(x, y, z)$

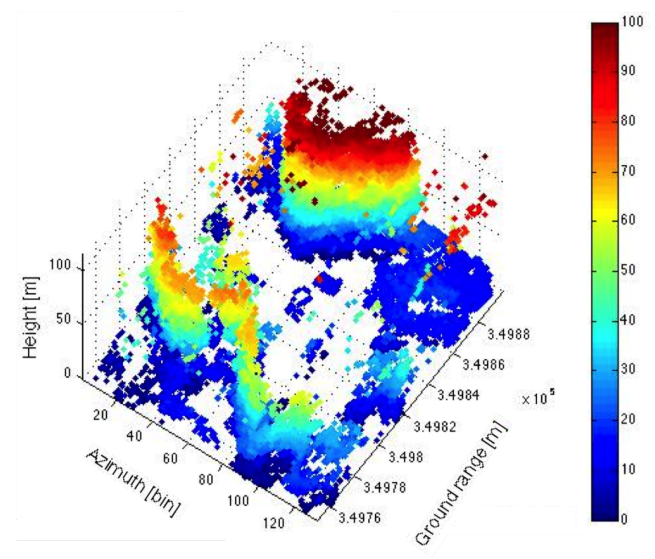

(a) 3-D height map: dominant, second and third scatters

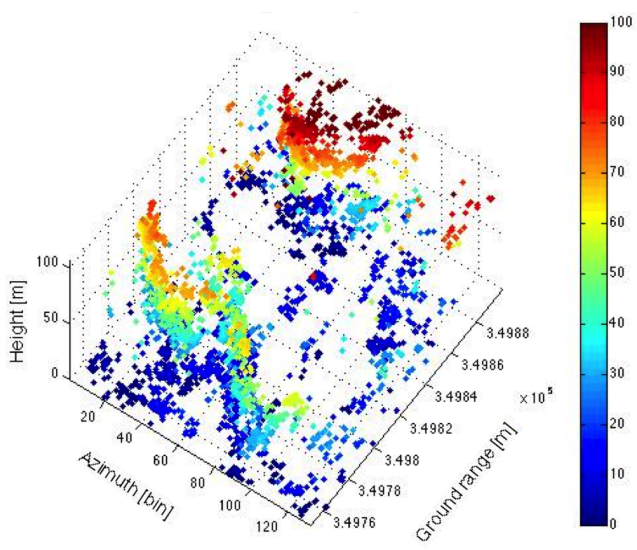

(b) 3-D height map: second detected scatters

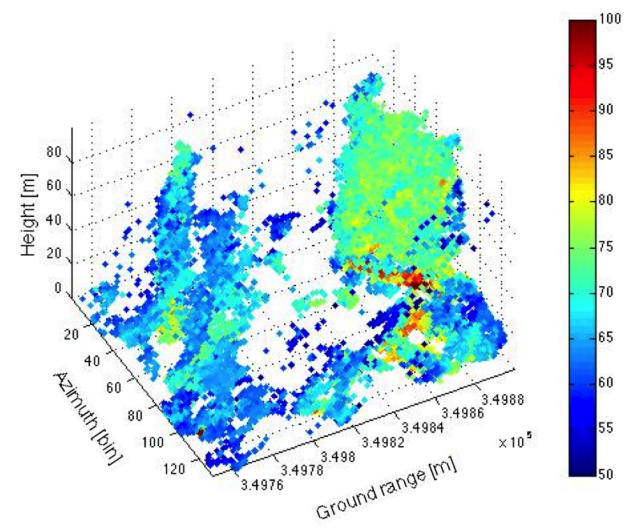

(c) 3-D reflectivity map: first three detected scatters

Figure 6. 3-D reconstructions over the AOI considering different number of scatters for each resolution cell using results derived by MUSIC approach

can then be measured using a modified coefficient of variation, $C V$, derived from $\left\{I_{i}(x, y, z)\right\}_{i=1}^{N_{i m}}$. The 3 -D reconstruction in Figure 9 displays the stable and unstable scatterers in term of the estimated $C V$ : unstable (yellow) pixels, i.e. points with high $C V$ values, lie mostly on the main skyscraper façades. In Figure 10 is represented an example of the time analysis aiming to extract the number and the indices of images that more perturb the 3-D temporally unstable scatterers: Figure 10(a) plots an extraction of the most perturbing image contributions varying the number of discarded images; Figure $10(\mathrm{~b})$ is a 3-D view of the image indices that more influence scatterers time 


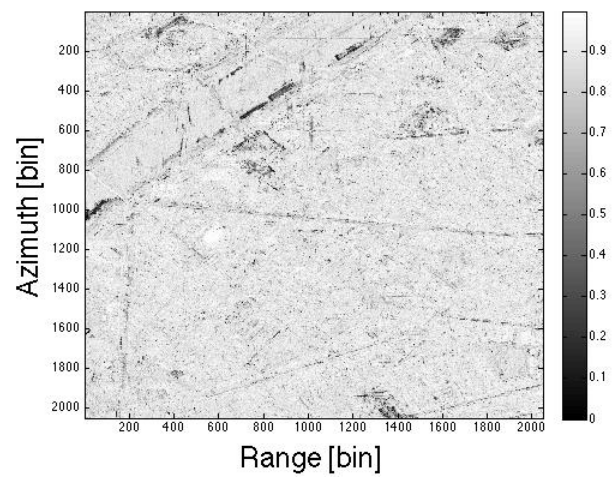

Figure 7. Image of the parameter $\Lambda$ on Paris test site, whose values go from 0 (very low stationarity) and 1 (very high stationarity)

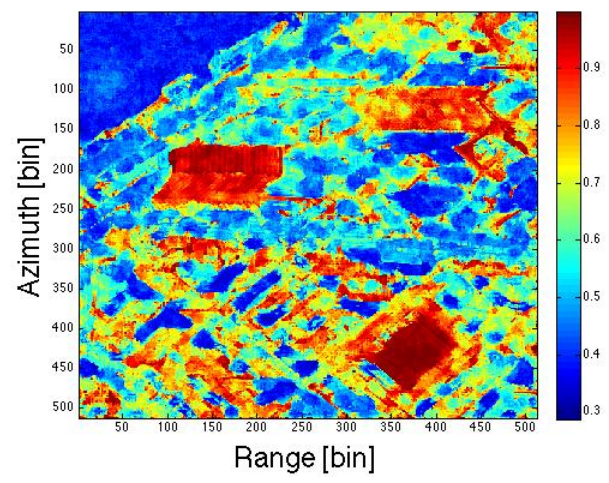

Figure 8. Coherence indicator trend over a subset ranges from 1 (high coherence) and 0 (low coherence)

stability at the first step (i.e. discarding one image).

\section{CONCLUSIONS}

Processing high resolution SAR data, such as ones acquired by TerraSAR-X, allows to achieve a strong improvement in 3-D imaging capabilities. At the same time, in urban areas analysis strong geometric distortions derived from layover have been taken into account.

In the present work several 3-D reconstructions about buildings hights, vertical reflectivity and time stability analysis have been carried out processing an adequate set of multitemporal images in order to perform a global characterization of build-up areas. We have demonstrated the potentiality of TomoSAR technique in distortions correction and determining information about the number of scatterers and the corresponding reflectivity within one resolution cell using basic mono-dimensional estimators as Beamforming, Capon and MUSIC.

\section{ACKNOWLEDGMENT}

The authors would like to acknowledge the German Aerospace Centre (DLR) for providing TerraSAR-X data used in this work in the frame of the project ID LAN1746.

\section{REFERENCES}

[1] A. Reigber and A. Moreira, "First demonstration of airborne SAR tomography using multibaseline L-band data," IEEE Transactions on Geoscience and Remote Sensing, vol. 38, no. 5 I, pp. 2142-2152, 2000.

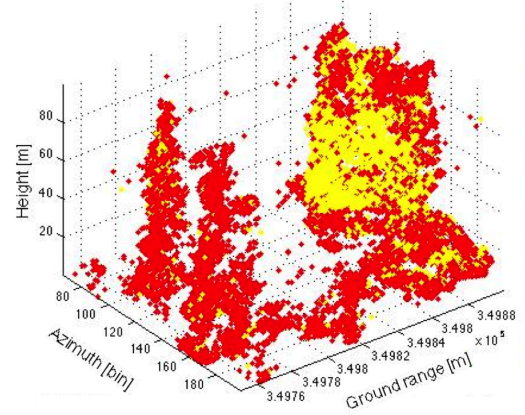

Figure 9. 3-D reconstruction over the AOI of the time stable (red) and unstable (yellow) pixels

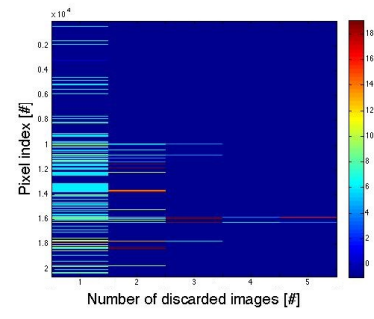

(a)

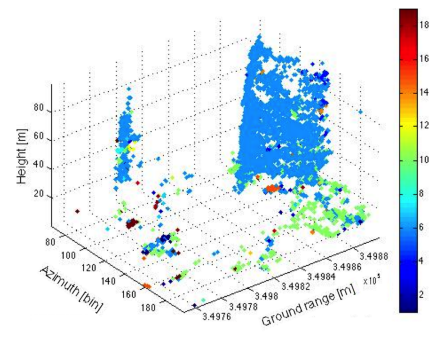

(b)
Figure 10. Extraction of the most perturbing contributions (left) and 3-D plot of the relative indices at the first step (right) over 3-D temporally unstable scatterers

[2] D. Reale, G. Fornaro, A. Pauciullo, X. Zhu, and R. Bamler, "Tomographic imaging and monitoring of buildings with very high resolution SAR data," IEEE Geoscience and Remote Sensing Letters, vol. 8, no. 4, pp. 661-665, 2011.

[3] G. Fornaro and F. Serafino, "Imaging of single and double scatterers in Urban areas via SAR tomography," IEEE Transactions on Geoscience and Remote Sensing, vol. 44, no. 12, pp. 3497-3505, 2006.

[4] P. Stoica and R. Moses, Spectral Analysis of Signals. Prentice Hall, 2005.

[5] F. Gini and F. Lombardini, "Multibaseline cross-track SAR interferometry: A signal processing perspective," IEEE Aerospace and Electronic Systems Magazine, vol. 20, no. 8 II, pp. 71-92, 2005.

[6] Y. Huang and L. Ferro-Famil, "3-D characterization of buildings in a dense urban environment using L-band pol-insar data with irregular baselines," vol. 3, 2009, pp. III29-III32.

[7] A. Budillon, A. Evangelista, and G. Schirinzi, "Three-dimensional SAR focusing from multipass signals using compressive sampling," IEEE Transactions on Geoscience and Remote Sensing, vol. 49, no. 1 PART 2, pp. 488-499, 2011.

[8] L. Ferro-Famil, Y. Huang, and E. Pottier, "Principles and applications of polarimetric SAR tomography for the characterization of complex environments," International Association of Geodesy Symposia, in press.

[9] X. Zhu and R. Bamler, "Tomographic SAR inversion by L1-norm regularization-the compressive sensing approach," IEEE Transactions on Geoscience and Remote Sensing, vol. 48, no. 10, pp. 3839-3846, 2010.

[10] _ "Demonstration of super-resolution for tomographic SAR imaging in urban environment," IEEE Transactions on Geoscience and Remote Sensing, vol. 50, no. 8, pp. 3150-3157, 2012.

[11] L. Ferro-Famil and E. Pottier, "Urban area remote sensing from L-band PolSAR data using time-frequency techniques," 2007.

[12] C. Hu, L. Ferro-Famil, and G. Kuang, "Ship discrimination using polarimetric SAR data and coherent time-frequency analysis," Remote Sensing, vol. 5, no. 12, pp. 6899-6920, 2013. 